\title{
El fin del sinhogarismo en Euskadi ¿mito o realidad?
}

\section{Miguel Angel Navarro Lashayas}

Plataforma BesteBi

<miwelnavarro@euskaltel.net>

Europar Batasunean abian da kalean lo egiten duten pertsonen gizarte-bazterketaren muturreko fenomenoa errotik kentzeko estrategia bat. Erronka garrantzitsua da, eta bere lorpenerako politika integralak ditu ezinbesteko. Beste herrialde batzuetan garatu diren esperientziak eta Euskadiko errealitatea kontuan hartuz, artikulu honetan bideratzen da etxegabekoen fenomenoari aurre egiteko proposamen integral bat. Hori dela-eta, aztertzen dira Euskadiko etxegabeko egoeran bizi diren pertsonen inguruko daturik eguneratuenak, sendotasuna duten alderdiak eta politika-mailan egin beharko liratekeen aldaketa eta osagai berriak, adibidez, helburua ahalbidetzen lagundu dezaketen etxebizitza, osasun, enplegu edo gutxiengoko errenten politikak. Betiere eskubideen ikuspegi bati helduz eta eskuartzearen erdiz erdiko ardatza pertsonaren duintasuna dela ziurtatuz.

\section{HITZ-GAKOAK:}

Etxegabeko pertsonak, gizarte-politikak, gizartebazterketa, gizarte-babesa, gizarte eskuartzeak.
Existe en la Unión Europea una estrategia para erradicar la cara más extrema de la exclusión social: las personas que duermen en la calle. Se trata de un reto importante, que requiere de políticas integrales para su consecución. Partiendo de las experiencias desarrolladas en otros países y de la realidad existente en el País Vasco, este artículo hace una propuesta integral para acabar con el sinhogarismo. Para ello, analiza los datos más actuales sobre las personas sin hogar en Euskadi, algunas de las fortalezas de que se disponen, y los cambios y elementos nuevos que habría que introducir en políticas como las de vivienda, sanidad, empleo 0 rentas mínimas para hacer factible el objetivo. Todo ello partiendo desde un enfoque de derechos, donde la dignidad de la persona debe ser el eje central de la intervención.

\section{Palabras Clave:}

Personas sin hogar, políticas sociales, exclusión social, protección social, intervención social. 


\section{El contexto europeo y estatal}

En 2010, el Parlamento Europeo aprobó una propuesta para acabar con el sinhogarismo en 2015: Se trata de la Declaración 61/2010, de 16 de diciembre de 2010. El objetivo es terminar con la expresión más visible del sinhogarismo, que son las personas que duermen en la calle. Para ello, se pide a la Comisión Europea que desarrolle una ambiciosa estrategia para abordar la situación de las personas sin hogar, y que ayude a los Estados miembros a que hagan lo propio a escala nacional, con arreglo a las orientaciones del informe conjunto de Protección Social e Inclusión Social, aprobado en marzo de 2010 como parte de la Estrategia UE 2020. En esta última, se establecen las prioridades para la próxima década, incluyendo cinco objetivos comunes que deben guiar la acción de los Gobiernos nacionales de la Unión Europea: la promoción del empleo; la mejora de las condiciones para la innovación, la investigación y el desarrollo; el abordaje del cambio climático; la mejora de los niveles de educación; y la promoción de la inclusión social, en particular, a través de la reducción de la pobreza. Para esto último, la Unión Europea se marca como objetivo sacar a veinte millones de personas del riesgo de pobreza y exclusión.

Son varios los países que ya tienen una estrategia nacional para cumplir estos objetivos (Dinamarca, Finlandia, Francia, Irlanda, Países Bajos, Portugal, Suecia y el Reino Unido), y otros, pese a no poseer todavía una estrategia nacional, han comenzado a informar de los avances producidos tras algunas medidas tomadas (Bélgica, Bulgaria, Luxemburgo, Polonia). El Estado español no se encuentra en ninguno de los dos grupos. Desde el tercer sector, se está apoyando dicha estrategia con el desarrollo de propuestas, informes de situación y presión política. Para la Federación Europea de Asociaciones Nacionales que Trabajan con Personas sin Hogar (Feantsa), el sinhogarismo puede y debe erradicarse, no sólo administrarse. Para ello, propone una estrategia global que se apoya en una serie de objetivos clave, tales como ${ }^{1}$ :

- Que nadie duerma en la calle: nadie debe verse obligado a dormir en la calle por falta de servicios de calidad adaptados a sus necesidades.

- Que nadie viva en un alojamiento de emergencia por un período superior al necesario. En ningún caso los albergues deben convertirse en sustitutos de hogares reales.

- Que nadie resida en alojamientos temporales más de lo estrictamente necesario. Los albergues, centros de acogida y viviendas tuteladas deben concebirse como pasos hacia una solución estable de alojamiento a corto y medio plazo.

${ }^{1}$ Pueden consultarse los diferentes informes sobre la estrategia de la UE en 〈http://www.feantsa.org/spip.php?rubrique123\&lang=en〉, y los informes sobre la estrategia de cada país en <http://www.feantsa. org/spip.php?rubrique143\&lang=en>.
- Que nadie abandone una institución sin alternativa de alojamiento. Los jóvenes extutelados, los enfermos dados de alta en hospitales y las personas que terminan su condena son los colectivos más vulnerables, y deben recibir ayuda para evitar el proceso circular que va de la tutela institucional a convertirse en persona sin hogar y de nuevo regresar a la tutela.

- Que ningún joven termine sin hogar como consecuencia de la transición a la vida independiente. Se deben hacer más esfuerzos para ayudar a las personas jóvenes a emanciparse y facilitar su acceso a la vivienda.

A escala estatal, tomando como base las recomendaciones de Feantsa, la Federación de Asociaciones de Centros para la Integración y Ayuda de Marginados publicó un documento con diez propuestas para una estrategia de erradicación del sinhogarismo (Faciam, 2010), que pasan por un enfoque basado en el derecho a una vivienda digna; la coordinación adecuada entre los diversos departamentos, ministerios y corporaciones locales; la prevención de situaciones de sinhogarismo mediante la provisión de suficiente vivienda de alquiler y otros mecanismos que faciliten el acceso a una vivienda y el mantenimiento de ésta; la promoción del empleo; la provisión de diferentes modelos de alojamiento; la calidad de los servicios; la ruptura del aislamiento y la soledad; el fomento de la participación de las personas sin hogar; la introducción de nuevas formas de intervención con las mujeres sin hogar; y la participación de todos los actores implicados en la elaboración y evaluación de las políticas de lucha contra el sinhogarismo.

Siguiendo la misma línea, la Federación de Entidades de Apoyo a las Personas sin Hogar (fePsh) publicó antes de las elecciones generales de 2011 una propuesta dirigida a los partidos políticos para la elaboración de una estrategia estatal de prevención y erradicación del sinhogarismo. Incluía medidas en torno a la prevención y la intervención, el trabajo en calle, el ejercicio de los derechos fundamentales, el modelo de alojamiento, la red de atención, y la promoción de políticas de alquiler de vivienda y vivienda protegida. Por último, las diferentes campañas anuales en torno al día de las personas sin hogar se han hecho eco de la estrategia europea y han planteado cinco propuestas en otros tantos años:

- 2010: coordinación y creación de un pacto de Estado por la inclusión social.

- 2011: no exclusión del Estado de derecho de las personas sin hogar indocumentadas.

- 2012: creación de un sistema de ingresos mínimos garantizados como derecho subjetivo de toda persona.

- 2013: garantía de un sistema de salud universal, gratuito y accesible para todos.

- 2014: ratificación, por parte del Estado español, de la Carta Social Europea Revisada de 1996, así como del Protocolo Adicional, por el que se 
establece el Sistema de Reclamaciones Colectivas de 1995 .

- 2015: último año de la campaña europea 'Acabar con el sinhogarismo es posible: nadie durmiendo en la calle'.

El objetivo de este artículo es realizar una propuesta de base para acabar con el sinhogarismo en Euskadi, partiendo de sus peculiaridades y teniendo en cuenta tanto las experiencias de otras regiones europeas en la materia, como las diferentes recomendaciones presentadas.

\section{El fenómeno del sinhogarismo en Euskadi}

Hasta hace poco, los únicos datos de los que se disponían en relación a las personas sin hogar en Euskadi eran los que resultaban de la Encuesta sobre las Personas sin Hogar del Eustat. Acaban de ser publicados los datos de 2012 y los anteriores corresponden a 2005, un lapso de tiempo demasiado largo como para permitir la planificación y seguimiento de una estrategia de erradicación del sinhogarismo. Por otra parte, se trata de una encuesta que recoge los datos de las personas que utilizan los servicios para personas sin hogar, pero no de aquellas que no acuden a los servicios; una encuesta con preguntas básicas que nos da un panorama general de la situación, condición necesaria, pero no suficiente, para profundizar en el tema. La Tabla 1 muestra algunos de los datos básicos de esta encuesta.

Tabla 1. Datos básicos de las personas sin hogar. Euskadi, 2005 y 2012

\begin{tabular}{|c|c|c|c|}
\hline & \multirow{3}{*}{$\begin{array}{c}2012 \\
328\end{array}$} & \multirow{3}{*}{$\begin{array}{r}2005 \\
508\end{array}$} \\
\hline & & & \\
\hline \multirow{4}{*}{ Territorio } & Araba & & \\
\hline & Bizkaia & 1.139 & 840 \\
\hline & Gipuzkoa & 623 & 486 \\
\hline & Euskadi & 2.090 & 1.833 \\
\hline \multirow{4}{*}{ Municipio } & Bilbao & 1.057 & 787 \\
\hline & Donostia-San Sebastián & 393 & 360 \\
\hline & Vitoria-Gasteiz & 328 & 508 \\
\hline & Resto de municipios & 311 & 178 \\
\hline \multirow{2}{*}{ Sexo } & Hombre & 1.701 & 1.382 \\
\hline & Mujer & 389 & 451 \\
\hline \multirow{4}{*}{ Edad } & 18-29 años & 724 & 521 \\
\hline & 30-44 años & 679 & 748 \\
\hline & 45-64 años & 639 & 512 \\
\hline & $\geq 65$ años & 48 & 52 \\
\hline \multirow{2}{*}{ Nacionalidad } & Española & 833 & 954 \\
\hline & Extranjera & 1.256 & 879 \\
\hline
\end{tabular}

Fuente: Encuesta sobre las Personas sin Hogar (Eustat, 2005 y 2012a).
Como vemos, los datos de personas sin hogar en Euskadi no sólo no han descendido, sino que se han incrementado ligeramente, tendencia condicionada, sin duda, por el aumento del colectivo de personas extranjeras en situación de vulnerabilidad, que, con respecto a la encuesta de 2005 , aumentan en una proporción del 43\%. El número de jóvenes sin hogar, y el de personas sin hogar ubicadas en Bilbao y otros municipios fuera de las capitales vascas también aumentan con respecto a la encuesta de 2005.

Además de las encuestas sobre la población sin hogar, Eustat también elabora otras sobre los centros destinados a este colectivo, la Estadística de Recursos para Personas sin Hogar, para conocer el número de plazas disponibles, los tipos de servicios prestados o los presupuestos que manejan, entre otras cuestiones. La última se ha realizado este año y aporta datos interesantes para conocer cómo se está interviniendo con esta población. Una breve pincelada se presenta en la Tabla 2.

Tabla 2. Datos básicos de los centros para personas sin hogar. Euskadi, 2012

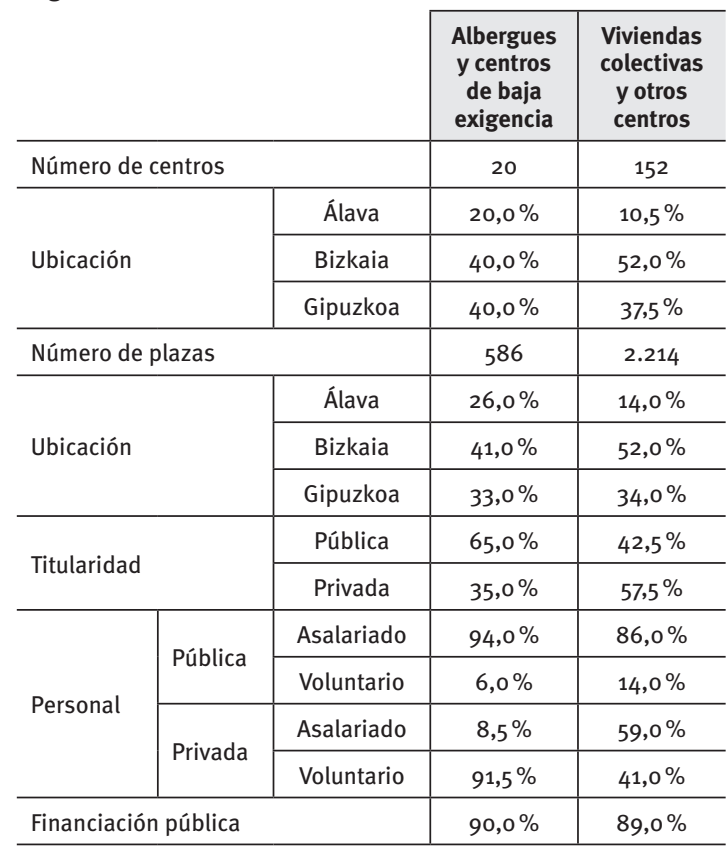

Fuente: Estadística de Recursos para Personas sin Hogar (Eustat, 2012b).

Casi la mitad de las plazas disponibles en alojamientos se encuentran en Bizkaia, lo que concuerda con los datos de número de personas sin hogar presentados anteriormente. Los albergues y centros de baja exigencia son, en su mayoría, de titularidad pública; en cambio, cuando se habla de viviendas colectivas y otros centros, la mayoría son de titularidad privada, aunque en cualquier caso, es la financiación pública la que sostiene la inmensa mayoría de ellos. Por último, existe una dependencia importante del voluntariado en la mayoría de los centros (salvo en los públicos), y a este respecto, falta quizá una mayor profesionalización en la atención a este colectivo, 
que, por otra parte, presenta especiales dificultades de intervención. En cuanto al tipo de prestaciones ofertadas por los centros, destacan las que tienen que ver con las necesidades básicas (restauración, alojamiento, información y acogida, orientación y derivación), mientras que la intervención en calle, los talleres de inserción y ocupacionales, los cuidados médicos, y las actividades artísticas y culturales son las menos ofertadas.

Como decíamos, hasta hace poco, los datos estadísticos sobre las personas sin hogar venían tan sólo del Eustat, y los referidos a toda España, del INE. En los últimos años, sin embargo, han surgido iniciativas desde las propias entidades sociales, en coordinación con universidades e instituciones públicas, para conocer el número y problemática de las personas que duermen en la calle. Se trata de datos más veraces que los recogidos en las estadísticas oficiales, ya que incluyen a las personas que no hacen uso de los recursos, y permiten conocer el número de las que se encuentran durmiendo literalmente en la calle, gracias a la colaboración de cientos de voluntarios que recorren las calles y recursos disponibles en una noche concreta. La primera ciudad que realizó un recuento nocturno fue Madrid, seguida de Barcelona (Cabrera, Rubio y Blasco, 2008). En Bilbao, se realizó el primero en 2010 (Comisión Onartu, 2011), y en Donostia-San Sebastián, en 2011 (SIIS Centro de Documentación y Estudios, 2011). En ambos casos, se utilizó la Tipología Europea del Sinhogarismo y la Exclusión Residencial (European Typology on Homelessness, ETHOS) [Feantsa, 2005] para categorizar el tipo de exclusión residencial que sufrían las personas encuestadas. El último avance ha sido la realización de un recuento nocturno conjunto de las tres capitales vascas en 2013 (SIIS, 2013). Poco a poco se avanza en un mayor conocimiento del problema y en una mayor coordinación entre territorios, instituciones y organizaciones sociales.

Si comparamos los datos del último recuento nocturno en las capitales vascas (2013) con los correspondientes a estas ciudades en la encuesta del Eustat (2012a), vemos diferencias importantes (Tabla 3 ).
En las tres ciudades, las cifras del Eustat y el recuento nocturno son parecidas, aunque son menores en los recuentos nocturnos. Tanto en Bilbao como en Donostia-San Sebastián, el recuento nocturno muestra una mayor proporción de personas extranjeras, fundamentalmente en esta última, con una diferencia de diez puntos porcentuales. También se advierte una población menos cronificada en los recuentos nocturnos. Las diferencias pueden obedecer a la mayor proporción de personas extranjeras que, de media, llevan menos tiempo sin hogar, y también a incluirse en las encuestas tanto a las personas que hacen uso de los recursos disponibles como a las que no.

A los estudios presentados hay que añadir algún otro que aporta información nueva sobre el fenómeno, como el realizado en Bilbao, donde se diferencian perfiles dentro de las personas sin hogar (Aierdi et al., 2009), o una reciente tesis que se ha realizado sobre los efectos psicosociales del sinhogarismo en la capital vizcaína (Navarro, 2013).

\section{La estructura de oportunidad}

Tras la presentación de los datos disponibles, la pregunta ineludible es ¿se puede acabar con el sinhogarismo en Euskadi? Para responderla, es necesario analizar si existe una estructura de oportunidad que permita el objetivo, esto es, si existen elementos dentro del sistema político y el entramado institucional que favorezcan su consecución. La posición de partida no es excesivamente negativa y se cuenta con algunos elementos, podríamos denominar fortalezas, que es necesario poner en juego, junto a otros nuevos, si queremos articular una política seria que permita erradicar el sinhogarismo. Los más importantes son:

- Las diversas leyes contra la exclusión social y los planes de inclusión, que han ido creado un corpus teórico y práctico sobre el tema, y permitido avances significativos en las últimas décadas.

Tabla 3. Número de personas sin hogar, procedencia y tiempo sin alojamiento. Bilbao, Donostia-San Sebastián y VitoriaGasteiz, c. 2012

\begin{tabular}{|c|c|c|c|c|c|c|c|}
\hline & & \multicolumn{3}{|c|}{ Eustat 2012} & \multicolumn{3}{|c|}{ Recuento nocturno 2013} \\
\hline & & Bilbao & $\begin{array}{c}\text { Donostia- } \\
\text { San Sebastián }\end{array}$ & $\begin{array}{l}\text { Vitoria- } \\
\text { Gasteiz }\end{array}$ & Bilbao & $\begin{array}{l}\text { Donostia-San } \\
\text { Sebastián }\end{array}$ & $\begin{array}{l}\text { Vitoria- } \\
\text { Gasteiz }\end{array}$ \\
\hline \multicolumn{2}{|c|}{ Número de personas sin hogar } & 1.057 & 393 & 328 & $850^{*}$ & $370^{*}$ & $310^{*}$ \\
\hline \multirow{2}{*}{ Procedencia } & Nacional & $42,0 \%$ & $42,5 \%$ & $44,2 \%$ & $37,1 \%^{\star \star \star}$ & $31,5 \%$ ** & $48,7 \%$ ** \\
\hline & Extranjero & $58,0 \%$ & $57,5 \%$ & $55,8 \%$ & $62,9 \%$ ** & $68,5 \%$ ** & $51,3 \%$ \%* \\
\hline \multirow{3}{*}{$\begin{array}{l}\text { Tiempo sin } \\
\text { alojamiento }\end{array}$} & 0.5 meses & $16,5 \%$ & $21,5 \%$ & $18,3 \%$ & \multicolumn{3}{|c|}{$19,9 \%$ \%** } \\
\hline & 6-11 meses & $10,0 \%$ & $10,0 \%$ & $12,0 \%$ & \multicolumn{3}{|c|}{$12,7 \%$ \%** } \\
\hline & $>1$ año & $73,5 \%$ & $68,5 \%$ & $69,7 \%$ & \multicolumn{3}{|c|}{ 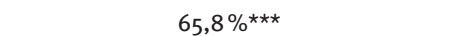 } \\
\hline
\end{tabular}

* Estas cifras incluyen las estimaciones más bajas del número personas que se encontraban durmiendo en la calle y no fueron localizadas en el recuento.

** Datos de la provincia.

$\star \star \star$ Datos de la CAPV.

Fuente: Elaboración propia a partir de la Encuesta sobre las Personas sin Hogar (Eustat, 2012a) y los recuentos nocturnos de las tres capitales vascas (SIIS, 2013). 
- La Ley de Servicios Sociales (12/2008), que surge del consenso de todos los grupos políticos, cuyo modelo de atención e intervención tiene carácter comunitario y coloca a los servicios sociales de base no sólo como punto de acceso al sistema, sino también con responsabilidades en la atención de necesidades de protección, autonomía e inclusión social. Es cierto que la ley no tiene desarrollados sus principales instrumentos, que son la Cartera de Prestaciones y Servicios y el Mapa de Servicios Sociales, pero ello es una oportunidad para que su concreción en el futuro tenga en cuenta la atención eficaz a las personas sin hogar.

- La fuerte red de entidades sociales con amplia experiencia en la materia y una cada vez mayor coordinación entre ellas. A ello se le unen algunas iniciativas interesantes, como la creación de plataformas que reivindican los derechos de las personas sin hogar y permiten situar el debate en un plano político, más allá de la mera atención asistencialista ${ }^{2}$.

- Las políticas de rentas mínimas, como la renta de garantía de ingresos y las ayudas especiales para la inclusión social, con un cierto recorrido histórico y aceptación social e institucional, que, pese a encontrarse en franco retroceso en los últimos años (con limitaciones en el acceso y recortes de las prestaciones), siguen siendo una herramienta esencial para la lucha contra la exclusión social.

- El servicio público de vivienda (Etxebide), con un importante parque de vivienda pública construida y experiencia en la intermediación en el mercado libre de alquiler (programa Bizigune).

- Un entramado institucional complejo, pero con cierta autonomía -incluso legislativa- con respecto al Gobierno central, que facilita la adopción de políticas más acordes a la realidad social concreta de Euskadi.

- Dispositivos de generación y avance del conocimiento, tales como el SIIS Centro de Documentación y Estudios, la revista Zerbitzuan, el Observatorio de Servicios Sociales de Álava, el Observatorio Social de Gipuzkoa y los diferentes observatorios del Gobierno Vasco (de vivienda, infancia y adolescencia, familia, servicios sociales, inmigración, juventud, drogodependencias).

- Un servicio público de salud de calidad (Osakidetza), con profesionales altamente cualificados.

A pesar de que los aspectos señalados son necesarios, ni por sí solos ni por su actual forma de funcionar son suficientes para erradicar el sinhogarismo. Es necesario introducir variaciones importantes y elementos nuevos para lograr el objetivo. Teniendo en cuenta las experiencias que se están desarrollando en Europa y aprovechando lo que ya existe, se proponen una serie de pilares fundamentales, sobre

2 Un ejemplo es la creación de la Plataforma BesteBi («http:// www.bestebi.net/১) en la ciudad de Bilbao. los que tendrían que sustentarse las políticas futuras en Euskadi para que nadie durmiera en la calle.

\section{Propuesta de actuación}

Es necesario apuntar que esta propuesta implica cambios profundos en políticas que no están dirigidas exclusivamente a personas en situación de exclusión social, ya que "los grupos excluidos no tienen interés únicamente por sí mismos en tanto elementos raros, extraños, diferentes o atípicos, sino que resulta fundamental investigar desde ellos para entender el mundo integrado y exclusivo de los que se encuentran instalados y acomodados socialmente" (Cabrera, 1998: 156). En consecuencia, resulta importante investigar qué ocurre en el extremo de la sociedad, porque nos suministra información de cómo funciona el centro - no son sino las dos caras de la misma moneda-. Por tanto, si queremos acabar con la exclusión social más grave, y no sólo dedicarnos a gestionar el problema, debemos caminar inevitablemente hacia transformaciones profundas en el seno de la sociedad, para no dejar de lado sistemáticamente todo un sector de la población.

En la Figura 1 se presenta de manera esquemática una propuesta de política integral para, posteriormente, analizar en profundidad cada uno de los aspectos señalados.

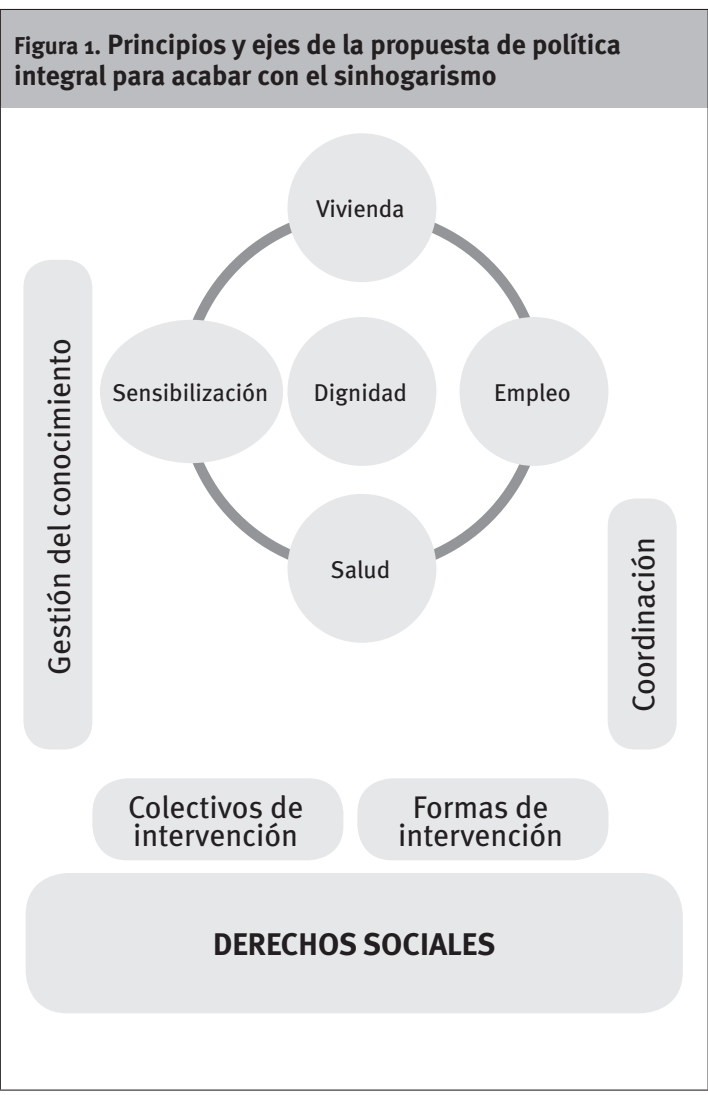

Fuente: Elaboración propia. 


\subsection{Derechos sociales}

La base sobre la que se debe sustentar cualquier política que quiera erradicar el sinhogarismo es la de la consecución de los derechos económicos, sociales y culturales a través del derecho a un nivel de vida adecuado (artículo 25 de la declaración Universal de los Derechos Humanos), que asiste a toda persona. El enfoque basado en derechos no es algo nuevo. En Euskadi, la Ley 12/1998 contra la Exclusión Social define este fenómeno como:

la imposibilidad de ejercer los derechos sociales, fundamentalmente el derecho al trabajo, pero también el derecho a la educación, a la formación, a la cultura, a la salud, a una vivienda digna, a la protección social. Desde esta perspectiva cabe considerar que está excluida toda persona que, en mayor o menor medida, se encuentra incapacitada o imposibilitada para el ejercicio de alguno o varios de los derechos sociales que otorgan estatus de ciudadanía a un individuo, es decir, toda persona que carece de los recursos personales sociales o económicos suficientes para ejercer estos derechos (exposición de motivos, artículo I).

Sin embargo, lejos de garantizar la consecución de dichos derechos, se niega su existencia (como el derecho a la vivienda) y se restringen los ya logrados (como el derecho a la salud), lo que afecta de manera especial a los colectivos más vulnerables. El Comité de Derechos Económicos, Sociales y Culturales (CDESC), órgano encargado de realizar el seguimiento al Pacto Internacional de Derechos Económicos, Sociales y Culturales (PIDESC), en su último informe del avance del Estado español en la materia (2004), se muestra preocupado por “la situación precaria de los numerosos inmigrantes, que sólo gozan de una protección limitada de sus derechos económicos, sociales y culturales" (punto 7). También pide que se "intensifiquen las actividades [...] a fin de dar asistencia a las personas sin hogar" (punto 38).

\subsection{Colectivos de intervención}

A pesar de que el desarrollo de las políticas que hagan efectivos los derechos económicos, sociales y culturales son comunes a toda la población, existen una serie de colectivos cuya especial vulnerabilidad recomienda políticas específicas que garanticen la igualdad de oportunidades en el acceso a tales derechos. Se trata de un tema sensible, ya que la población puede sentir que se da un trato prioritario o de favor a algunos sectores, pero tampoco podemos permitir que una falsa uniformidad oculte significativas situaciones sociales de desigualdad, que en la práctica impiden el acceso a derechos y perpetúan la injusticia. Algunos de los grupos más vulnerables son:

- Menores y jóvenes extranjeros no acompañados: en los últimos años, se ha dado un rejuvenecimiento del colectivo de personas sin hogar, algunas de las cuales provienen del sistema de protección de menores. En cualquier caso, se trata de adolescentes y jóvenes con escasa formación y experiencia laboral, alta estigmatización social y problemáticas comunes a gente de su edad, para quienes la calle puede producir una especial desestructuración de su personalidad.

- Expresidiarios: la salida de la cárcel ha sido señalada como una de las experiencias que pueden llevar a una persona al sinhogarismo (Fitzpatrick, Kemp y Klinker, 2000). Para algunos investigadores, el mayor proveedor de alojamiento para las personas sin hogar es el sistema penitenciario. En el estudio realizado en Bizkaia (Aierdi et al., 2009), un $38 \%$ de los encuestados habían estado en la cárcel, y un $12 \%$, en un centro de internamiento de menores.

- Inmigrantes y demandantes de asilo: son el colectivo de personas sin hogar de mayor crecimiento en toda Europa, y los datos, tanto de los recuentos nocturnos como de la encuesta del Eustat (2012a), muestran que son mayoría. La especial vulnerabilidad de las personas que se encuentran en situación irregular y de aquellas cuya demanda de asilo ha sido denegada o no admitida a trámite merece especial consideración. El 43,6\% de las personas extranjeras encuestadas en DonostiaSan Sebastián (2011) y el $72 \%$ en Bilbao (2010) se encuentran en situación irregular.

- Mujeres y personas mayores: son dos colectivos que aumentan su presencia en la calle, aunque no de manera tan notoria como las personas extranjeras. En el caso de las mujeres, se da una invisibilización debido, en parte, al apoyo de redes sociales y a la prevalencia de casos de trata y prostitución encubierta. Las mujeres solas con cargas familiares son un colectivo para el que los recursos sociales apenas pueden dar respuestas adecuadas. Algo similar ocurre con las personas mayores, cuya presencia en la calle es menos notoria, pero que según la tipología ETHOS sufren de manera significativa situaciones conceptualizadas como vivienda insegura y vivienda inadecuada.

\subsection{Formas de intervención}

El punto de partida de la intervención debe consistir en recuperar la responsabilidad pública en la lucha contra el sinhogarismo. Según la última encuesta del Eustat (2012b), casi el 60\% de los centros que trabajan con personas sin hogar son de titularidad privada. Los centros y servicios de atención a personas sin hogar deben utilizar las estructuras de los servicios públicos, aunque se puedan establecer actuaciones específicas orientadas a facilitar el acceso a las estructuras, prestaciones y servicios normalizados, todo ello dando protagonismo a lo local. La Ley de Servicios Sociales (Ley 12/2008) es una oportunidad para ello, porque aboga por un enfoque comunitario, lo que significa que los servicios sociales de atención primaria deben dirigir su labor hacia la dinamización y desarrollo de la comunidad allí donde se encuen- 
tran. Ya existen algunas propuestas de cómo debería ser el nuevo rol de los servicios sociales en Euskadi (Duque, 2012).

Además del protagonismo de lo público y la integración en servicios normalizados siempre que sea posible, hay otros dos aspectos de la intervención que hay que destacar: el fomento de la prevención y la participación de las personas usuarias. Para lo primero, son especialmente relevantes las políticas de vivienda, que trataremos más adelante, así como la rápida intervención una vez la persona se queda en la calle, que debe procurar que la estancia en la calle sea lo más breve posible, para evitar su cronificación y las dificultades de intervención asociadas a ella. En cuanto a lo segundo, se trata de una preocupación a escala europea que ha suscitado diversas investigaciones ${ }^{3}$. La participación implica cambios en los dispositivos de atención que permitan a las personas tomar decisiones sobre su propia vida y sobre los servicios que utilizan. Se debe favorecer las decisiones conjuntas y la implicación efectiva de las personas en el funcionamiento del servicio, asumiendo responsabilidades en determinadas tareas. Se trata de un proceso que en muchos casos se define con la metáfora de los escalones, que van de la información a la consulta, las decisiones conjuntas, el trabajo en equipo y, por último, al apoyo a las iniciativas independientes.

En un metanálisis realizado recientemente sobre los efectos psicológicos de la exclusión social (Blackhart et al., 2009), se llega a la conclusión de que genera aplanamiento afectivo o un estado de neutralidad emocional. Otros autores (Valverde, 2009) sostienen que la exclusión social es, ante todo, un proceso de despersonalización, de pérdida de identidad personal y de intimidad, y que la cronificación de la situación de sinhogarismo llega a modificar la identidad personal de quien la sufre, al perder toda esperanza de cambio (Navarro, 2013). La autoeficacia percibida, así como la orientación hacia el futuro han sido analizadas como condicionantes del comportamiento de las personas sin hogar (Epel, Bandura y Zimbardo, 1999). El fomento de la participación y las intervenciones encaminadas a que las personas recuperen el control sobre su propia vida son herramientas válidas para contrarrestar los efectos nombrados.

\subsection{Salud}

Las encuestas realizadas a las personas sin hogar muestran que la percepción de su salud es buena: tan sólo el $9 \%$ afirma que su salud es mala o muy mala (Eustat, 2012a). Sin embargo, la percepción subjetiva de buena salud contrasta con los datos objetivos. Por ejemplo, en la misma encuesta del Eustat, el 24,4\% de las personas entrevistadas declaran tener una enfermedad grave o crónica.

${ }^{3}$ En 2009, Feantsa designó la participación como tema prioritario de investigación. Al respecto, puede verse <http://www.feantsa.org/ spip.php?rubrique28\&lang=en〉.
En los recuentos nocturnos llevados a cabo en las ciudades de Madrid y Barcelona (Cabrera, Rubio y Blasco, 2008; Cabrera y Rubio, 2008), el 46\% de las personas entrevistadas padecen algún problema de salud. Su esperanza de vida es 25 años menor al resto de la población, y presentan entre 2 y 50 veces más problemas de salud físicos que la población en general (Muñoz, Vázquez y Vázquez, 2003). Los datos son consistentes con las investigaciones internacionales que muestran la tendencia a encontrar peores indicadores de salud entre los grupos más desfavorecidos económica y socialmente, especialmente entre las personas sin hogar (Rossi, 1989; Wright y Weber, 1987).

Además de peor salud, las personas sin hogar también tienen mayores dificultades para acceder a los sistemas de salud públicos, que en la mayoría de los casos no son capaces de adaptarse a la situación de dichas personas, ni dar respuesta a sus necesidades sociosanitarias (Feantsa, 2006). Se deben crear dispositivos de mediación y acompañamiento que permitan el acceso al sistema de salud público en igualdad de condiciones, así como facilitar los trámites administrativos para la obtención de la tarjeta sanitaria. Las condiciones de vida en la calle no son las más idóneas para conservar documentos y seguir el rigor administrativo. Por otra parte, no hay que olvidar que la mayoría de las personas que duermen en la calle son extranjeras, y una proporción importante de ellas no cuenta con permiso de residencia -en algunos casos, ni siquiera con pasaporte-. A pesar de que el Gobierno Vasco ha implementado medidas para contrarrestar el Real Decreto 1192/2012 4 , el acceso al sistema sanitario para dichas personas sigue siendo un escollo importante.

Uno de los temas que más investigación ha generado es el papel que juegan los problemas de salud mental y de abuso de sustancias en la etiología y mantenimiento de la situación sin hogar. Cuando se han analizado los procesos de inicio de la enfermedad mental antes, durante y después de la situación de sin hogar (Muñoz, Vázquez y Vázquez, 2003), se ha comprobado que la mayoría de las personas sufrían las primeras crisis de salud mental, o incluso de abuso de alcohol, antes de sufrir su primera situación sin hogar.

Los datos más sólidos acerca de la relación entre salud mental y personas sin hogar sitúan la prevalencia de los trastornos graves entre el $25 \%$ y el $35 \%$ (Robertson, 1992). En un metanálisis (Lehman y Cordray, 1993), se encontró que entre el $46 \%$ y el $49 \%$ de las personas $\sin$ hogar sufrían dependencia de drogas, y entre el $29 \%$ y el $31 \%$, abuso de alcohol. Otro metanálisis, realizado más recientemente (Fazel et al., 2008) a partir de los resultados de 29 trabajos y una muestra agregada de 5.684 personas sin hogar, muestra una gran heterogeneidad en los resultados, con tasas de dependencia

${ }_{4}^{4}$ Por el que se regula la condición de asegurado y de beneficiario a efectos de la asistencia sanitaria en España, con cargo a fondos públicos, a través del Sistema Nacional de Salud. 
del alcohol de entre el $8,5 \%$ y el $58,1 \%$ (37,9\% de media), tasa de dependencia de otras sustancias de entre el $4,7 \%$ y el $54,2 \%$ ( $24,4 \%$ de media), prevalencia de trastornos psicóticos de entre el $2,8 \%$ y el $42,3 \%$ ( $12,7 \%$ de media), una prevalencia media de depresión mayor del $11,4 \%$, y de trastornos de personalidad, del 23,1\%. La encuesta del Eustat (2012a) muestra un menor consumo de alcohol, con un 73,9\% de personas que declaran ser abstemias y tan sólo un $1,8 \%$ que declaran tener un consumo alto o excesivo de alcohol. En la última investigación realizada en Bizkaia (Aierdi et al., 2009), un $18 \%$ de las personas encuestadas consumen alcohol habitualmente, $y$ un $39 \%$, de vez en cuando.

Más allá del baile de cifras, la salud mental y el abuso de sustancias son variables que marcan claramente el devenir de las personas sin hogar, por el deterioro personal que provocan, su alta visibilidad social y el estigma asociado, y porque son escasos los recursos de intervención. En los últimos años, se han realizado avances en la atención en salud mental, con la creación de equipos específicos que intervienen fuera del ámbito hospitalario (Garay et al., 2012), aunque siguen faltando recursos de intervención en el terreno del abuso de sustancias.

El panorama descrito es tan sólo un reflejo de dos deficiencias históricas de Osakidetza: la atención integral a la salud mental y la creación de un espacio sociosanitario real. Recientemente, Feantsa ha publicado una serie de recomendaciones para el desarrollo de un sistema de asistencia social y salud integrado y coordinado (Feantsa, 2013), entre las que se encuentran:

- Diseñar servicios flexibles y adaptados para las personas sin hogar.

- Adaptar los modelos de atención para cubrir las necesidades de salud de las personas sin hogar.

- Aumentar la conciencia y comprensión de la naturaleza compleja e interdependiente de las necesidades de salud de estas personas entre los profesionales de la salud, a través de la formación.

- Mejorar los protocolos del alta hospitalaria para que nadie tenga que recuperarse de su enfermedad en la calle.

- Impulsar intervenciones de promoción de la salud y estrategias de prevención específicas para el colectivo.

- Desarrollar programas de reducción de daños, tales como Housing First, intercambio de jeringuillas, terapias de sustitución, espacios de consumo seguro y programas de bebida controlados.

- Promover la participación de las personas sin hogar en el diseño e implementación de intervenciones de salud.

- Fomentar la vida independiente mediante la prestación de apoyo adecuado para las personas en sus hogares, en función de sus necesidades.

Se debe poner en marcha un debate sobre el modelo de intervención en este ámbito. Además de anali- zar el asunto del espacio sociosanitario, se debe reflexionar sobre la baja exigencia y su encaje en los modelos de asistencia sanitaria tradicional, los nuevos modelos de tratamiento en salud mental, como el asertivo comunitario, y la siempre delicada cuestión del tratamiento involuntario.

\subsection{Empleo y rentas mínimas}

La encuesta sobre personas sin hogar del Eustat (2012a) muestra que un $74,5 \%$ se encuentran paradas y otro $11,5 \%$ son clasificadas como 'otros inactivos', siendo un 4,6\% las que tienen trabajo y el resto, personas que cobran pensiones de jubilación o invalidez.

Las personas sin hogar que afirman disponer de empleo declaran una variedad importante de ocupaciones, aunque casi todas tienen en común que se trata de ocupaciones marginales o precarias, tales como recoger chatarra, descargar camiones o recoger vasos en locales de hostelería. Pero no disponer de empleo no conlleva que no existan ingresos económicos. Las ayudas sociales (en el caso del País Vasco, fundamentalmente la renta de garantía de ingresos [RGI]) mitigan la exclusión, aunque su efecto no está claro. La encuesta del Eustat (2012a) muestra que tan sólo el $27,3 \%$ de las personas encuestadas no cuentan con ningún tipo de ingreso económico. Un 13\% recibe la RGI, menor proporción que los que reciben ayuda oenegés $(15,5 \%)$ y otro tipo de prestaciones $(16,5 \%)$.

Resulta llamativo que, en aquella situación que se puede considerar paradigmática de la exclusión social, como es el sinhogarismo, las prestaciones públicas creadas para paliar dichas situaciones tengan una incidencia tan baja. Los requisitos de acceso (Ley 4/2011), y en concreto algunos de ellos, como el tiempo de empadronamiento y la existencia de un contrato de alquiler o subarriendo de una vivienda, influyen en ello.

Se necesitan políticas activas de empleo que faciliten la inserción laboral de las personas empleables, pero también el apoyo para formación en los casos necesarios (de vital importancia para los jóvenes extranjeros que cuentan con baja cualificación y nula experiencia laboral) y el fomento del empleo protegido mediante empresas de inserción para los casos de baja o nula empleabilidad. Cada vez son más numerosas las empresas de inserción, se encuentran más coordinadas entre sí, con redes como Gizatea o Reas, y han diversificado los sectores donde intervienen, como artes gráficas, construcción, hostelería, limpieza y mantenimiento, medio ambiente, mensajería, montaje de estructuras, u ocio y cultura, entre otros. Las instituciones públicas deben apoyar dichas iniciativas e incluir en la contratación de sus servicios beneficios para ellas.

\subsection{Vivienda}

La falta de vivienda genera una situación de inseguridad, desarraigo y ausencia de intimidad, que conduce a procesos de gran deterioro personal y de las 
relaciones sociales. El acceso a una vivienda como espacio propio abre la posibilidad de reconstruir la confianza en uno mismo y la generación de participación social (Feantsa, 2008). El derecho a la vivienda es esencial para garantizar la vida digna de las personas. Sin embargo, es uno de los derechos sociales en los que se constata que no sólo no se ha avanzado en su universalización, sino que se ha retrocedido, siendo la situación de la vivienda un buen indicador del nivel de bienestar social (Ararteko, 2008).

Se podría esperar que, si hay personas sin hogar, es porque faltan viviendas donde alojarse y se debe lidiar con un mercado reducido de viviendas disponibles que, inevitablemente, dispara los precios por la competencia en el acceso. Nada más lejos de la realidad. La encuesta bienal sobre vivienda vacía en la CAPV (Observatorio Vasco de la Vivienda, 2012) calcula en unas 85.000 las viviendas vacías en Euskadi en 2011. Quitando las segundas residencias, encontramos un total de 58.711 viviendas con posibilidades de utilización. Teniendo en cuenta que la encuesta del Eustat (2012a) cifra en 2.090 las personas atendidas en los centros para personas 'sin hogar' en Euskadi, tenemos que por cada una de ellas hay 28 viviendas perfectamente alquilables (descartando segundas residencias) que podrían ser utilizadas. Los datos pueden ser todavía más escandalosos, porque según la Encuesta sobre Vivienda Vacía del Eustat (2012c), a finales de 2011 había 116.157 viviendas vacías, lo que casi duplica los datos manejados por el Observatorio Vasco de la Vivienda.

El mercado de vivienda está completamente vedado a las personas en situación de exclusión social. Las viviendas en venta libre tenían un precio medio de 3.653 euros $/ \mathrm{m}^{2}$ en el último cuatrimestre de $\mathbf{2 0 1 2}$, tras el descenso producido por el fin de la burbuja inmobiliaria, que, en diez años (de 1999 al 2009), elevó los precios de unos 1.400 euros $/ \mathrm{m}^{2}$ a los 4.100 euros $/ \mathrm{m}^{2}$, un incremento de casi el 300\%. Según el Observatorio Vasco de la Vivienda («http://euskadi.net/r41-ovhome/es〉), el precio medio de una vivienda libre en el País Vasco es de 332.000 euros, y el de una vivienda social, de 109.000 euros. A pesar de la diferencia considerable de precio, la vivienda social en venta está destinada a la clase media, ya que para afrontar dicho gasto es necesario pedir una hipoteca en un banco, cuya concesión va a depender en exclusiva de la solvencia económica demostrable.

Con la vivienda en venta, libre o de protección, vetada para las rentas más bajas, la única salida es el alquiler. Dos problemas importantes lastran esa posibilidad. El primero es el precio, que ascendía a una media de 856 euros/mes en el último trimestre de 2012. El segundo es la escasez de vivienda de alquiler. El último informe realizado por el Observatorio Vasco de Vivienda sobre el alquiler en el País Vasco (2013b), permite estimar que el $6,4 \%$ de los hogares vascos residen en una vivienda en régimen de alquiler, mientras que los hogares que han accedido a la propiedad de su vivienda habitual representan el $91,5 \%$.
Con los precios existentes en el mercado libre, las personas con menos recursos tan sólo pueden contar, como provisión real de alojamiento, con las viviendas de alquiler que tienen algún tipo de protección oficial. Aunque en los últimos años la proporción construida de este tipo de viviendas ha aumentado, su volumen sigue siendo escaso. La máxima proporción de vivienda de alquiler de protección construida se dio en 2008 , con un $36 \%$ del total de viviendas iniciadas con algún régimen de protección, pero fue un espejismo, ya que la media desde 2004 no llega al $20 \%$ y en 2012 fue tan sólo del 4,9\%. Por todo ello, el peso del alquiler protegido dentro del mercado de alquiler es del $37 \%$, incluyendo las viviendas del programa Bizigune (5.102 viviendas en 2012, aunque con escaso crecimiento desde 2008) [Observatorio Vasco de la Vivienda, 2013b].

Nos encontramos con un mercado libre que deja completamente fuera a una parte importante de la población (entre la que se encuentran las personas sin hogar), y un mercado de protección dependiente de las políticas del Estado orientado a la clase media, con baja proporción de viviendas de alquiler a precios asequibles (231 euros mensuales en las viviendas de alquiler con algún tipo de protección en el País Vasco), que son las únicas a las que podrían acceder las personas sin hogar. A ello se añade que no existe ningún tipo de política para facilitar el acceso a las personas sin hogar a una vivienda y que el enfoque de las intervenciones en este sentido sigue siendo meramente asistencialista, al considerar la vivienda como un paso final de una etapa de inserción y normalización en la sociedad, que permite a la persona acceder a una provisión de alojamiento a través de los circuitos considerados normalizados. Es por ello por lo que se debe optar por una política diferente.

El acceso a la vivienda de los sectores de la población más vulnerables nos va a dar la medida de las facilidades de acceso del resto de la población. Ello no quiere decir que las políticas deban ir encaminadas a un sector específico, pero a nadie se le escapa que, si la gente que está en peores condiciones tiene posibilidades de acceso a la vivienda, tanto más las tendrán quienes cobran un salario digno. Para lograrlo, la vivienda tiene que ser un derecho, hay que alejar la mirada de la posesión y centrarla en el acceso. Sobre dicha base se deben articular otra serie de medidas:

- Acceso a la vivienda: existe una Ley del Suelo y un Pacto Social por la Vivienda que nos permiten avanzar en cuestiones como el cambio del sistema de sorteos para adjudicar las viviendas, las medidas tributarias a favor del alquiler, el fomento de la rehabilitación y el establecimiento de precios diferenciados en función del nivel de renta. Sin embargo, no es suficiente; se necesita una Ley de Vivienda que siente las bases de una política distinta en la materia. En las dos últimas legislaturas ha habido otros tantos intentos que no han terminado de concretarse. Algunos aspectos que debería abordar una futura Ley de Vivienda son: 
- El derecho subjetivo a la vivienda reclamable judicialmente.

- Acabar con los coches de lujo en garajes de viviendas de protección oficial ('efecto BMWPO'): una vivienda nunca debe tocar por sorteo para el resto de la vida, independientemente de los progresos en el nivel de renta.

- La construcción de VPO para su posterior venta supone la liquidación de recursos públicos bajo una mirada cortoplacista. No sólo hay que fomentar el alquiler, sino que éste debe ser la opción preferente de Etxebide y el único fin de la construcción de vivienda nueva.

- Promover formas alternativas a la compra y el alquiler en el acceso a la vivienda. El Observatorio Vasco de la Vivienda ha publicado un informe (2013a) que recoge algunas iniciativas novedosas y dignas de tener en cuenta que se desarrollan en Europa y EE.UU. En todo caso, se debe acabar con figuras obsoletas que no aportan nada en política de vivienda, como las viviendas tasadas, a las que pueden acceder rentas que superan los 50.000 euros anuales.

- No se puede hablar de una política de vivienda sin acabar con las viviendas vacías. Los datos hablan por sí solos y se debe tomar ejemplo de las iniciativas desarrolladas en otros países europeos para evitar esta lacra. En Dinamarca, es obligatorio comunicar al Estado la existencia de una vivienda vacía, y si la persona propietaria la mantiene así más de seis meses, recibe una multa. Los Países Bajos permiten que una vivienda sea ocupada cuando se encuentra vacía durante más de un año. En Francia, se facilita el alquiler de vivienda vacía, y se puede requisar una vivienda durante doce años si se encuentra vacía dieciocho meses y la persona propietaria no ha hecho lo posible para facilitar su arrendamiento.

- Frenar los desahucios: según datos del Observatorio Vasco de la Vivienda, en 2012 tuvieron lugar 785 ejecuciones hipotecarias, de las que el $85 \%$ eran de primera vivienda. No se puede acabar con el sinhogarismo si se sigue desahuciando sin solución alguna a gente de su casa.

- Los modelos de alojamiento: además de las políticas de vivienda, se debe dar respuesta a la diversidad de situaciones que requieren un alojamiento, tanto de urgencia como unidades de media y larga estancia, alojamientos de transición hacia una vivienda independiente, recursos de baja intensidad, o alojamientos adaptados a unidades familiares o personas mayores. Entre la calle y la vivienda independiente, existe un amplio abanico de posibilidades que, en muchos casos, van a facilitar la transición hacia la vida independiente, y deben ser tenidas en cuenta. Mención especial merece el modelo conocido como Housing First o 'vivienda primero', que sostiene que el objetivo prioritario debe ser alojar a la persona en una vivienda tan pronto como sea posible. Tradicionalmente, la lógica utilizada es que se debe trabajar con la persona sin hogar en albergues y mediante programas de educación de calle para prepararla para la vida autónoma. Se hace hincapié en que sólo una vez que se solucionen, o mitiguen, cuestiones derivadas de problemas personales se puede esperar que la persona se reincorpore con éxito a una vivienda de carácter estable. Según el enfoque de la vivienda primero, muchos de estos problemas se pueden trabajar con mayor facilidad en el contexto de un alojamiento estable, por lo que primero se concede dicho alojamiento sin condición previa alguna. En países como Irlanda, Finlandia y Escocia, es un aspecto central de la política frente al sinhogarismo; en otros, como Francia, Reino Unido, Polonia y Alemania, se están desarrollando experiencias de este tipo. Ni en Euskadi ni en el Estado existen aún experiencias de Housing First, y sería importante impulsarlas.

\subsection{Sensibilización}

En un ensayo clásico, Edwin Goffman (1980) definió el estigma como un atributo personal negativo y en extremo denigrante, que hace a una persona diferente de las otras y de una clase indeseable o categoría inferior. Goffman aplicó el término de estigma negativo a cualquier condición, atributo, rasgo o comportamiento del portador como algo culturalmente inaceptable e inferior, que conlleva sentimientos de vergüenza, culpabilidad y humillación. El estigma no es un simple atributo individual, sino que es un proceso interpersonal, producto de las relaciones sociales: 'el otro' tiene un papel fundamental en la creación del estigma; su mirada, sus gestos e incluso sus palabras influyen en ello.

Algunas teorías explican la exclusión social en la negación de oportunidades que padecen grupos sociales estigmatizados, fundamentalmente a través de la discriminación activa y los prejuicios. Buen ejemplo de ello lo encontramos en una investigación realizada en Bilbao (SOS Racismo y CEAR-Euskadi, 2012), que mostraba que las personas extranjeras recibían, bajo las mismas condiciones, un $80 \%$ menos de ofertas de pisos de alquiler que las personas autóctonas. El estigma y la discriminación también tienen efectos en la identidad del individuo que los sufre, cuya autoestima y personalidad quedan afectadas de modo importante (Taylor, 1993), y puede ser un factor altamente deshumanizante y alienante (Navarro, 2013).

El colectivo de personas sin hogar sufre una importante estigmatización, provocada incluso por los propios investigadores sociales. Así, hasta bien entrados los años ochenta, los principales estudios en torno a las personas sin hogar se desarrollaron en los Estados Unidos, situándose en el ámbito de los problema sociales que centran su atención en las características de las personas y no en el 
componente estructural o social de la exclusión. Las principales investigaciones buscaban desenmascarar las características, el carácter y condiciones personales de esos sujetos extraños, ajenos a la sociedad, que poblaban algunos barrios de la ciudad. Autores como Bahr $(1968,1973)$ hablan de la desafiliación y desarraigo en que viven estas personas que cuentan con vínculos muy tenues con la familia y con pocos o ningún amigo. Para este autor, la persona sin hogar porta el estigma social de ser alguien defectuoso físicamente, mentalmente, moralmente (un pervertido, un criminal), psicológicamente (baja autoestima, elevada autoagresión), socialmente (desarraigado), legalmente (tratado por las autoridades como si no fuera un hombre libre), económicamente (empobrecido, desempleado) y ecológicamente (vive en un barrio donde ninguna persona decente querría vivir).

En el Estado español, la imagen de la persona sin hogar ha estado siempre ligada a la del 'caradura' que no quiere trabajar y 'tiene lo que se merece'. Ya en 1933 se promulgó la Ley de Vagos y Maleantes, que se mantuvo durante buena parte del Franquismo, continuada por la Ley de Peligrosidad Social de 1970, que incluía a los 'mendigos habituales'. La sociedad tiende a rechazar y mostrar indiferencia (cuando no desprecio) a las personas sin hogar; los prejuicios sociales ('son vagos', 'están en la calle porque quieren') impiden ver la dureza de la vida de estas personas, y obvian la responsabilidad de la ciudadanía y de las administraciones públicas frente a tales circunstancias. Ya hemos visto que la situación de los excluidos está ligada a la de aquellos ‘integrados’ en la sociedad: todos forman parte del mismo sistema que encumbra a unos, mientras que arroja a la periferia a otros, por lo que el rechazo y la indiferencia no son sino actitudes cómplices de la situación que vive dicha persona.

Se necesita una importante labor de sensibilización de la problemática de la exclusión social, de sus causas y sus consecuencias, pero también de la responsabilidad como sociedad de lo que sucede a nuestro alrededor. Se debe promover una auténtica conciencia de las causas estructurales de la exclusión que mitigue la visión de las personas sin hogar como seres extraños que no tienen nada que ver con el resto de la sociedad. Las personas sin hogar no forman parte de un submundo paralelo distinto al nuestro. Es importante situar su problemática dentro del propio funcionamiento social y como producto de él. Por tanto, los signos de la existencia de un mundo aparte dentro de las personas sin hogar deben ser tomados como una consecuencia, y no como una causa de su situación (Navarro, 2013).

Además de la sensibilización, hay que promover la instauración de políticas antidiscriminatorias, inexistentes en la actualidad. La publicitada Ley por la Igualdad de Trato a escala estatal no terminó de cuajar en la pasada legislatura y no tiene visos de que se vaya a producir en ésta. Desde las instancias autonómicas no se puede ser ajeno a la realidad de racismo y discriminación existente, y se debe impulsar una Ley vasca por la Igualdad de Trato y no Discriminación que mitigue sus efectos, a la espera de que en un futuro se pueda llegar a crear una ley a escala nacional. El III Plan de Inmigración Ciudadanía y Convivencia Intercultural del Gobierno Vasco marca, como una de sus líneas estratégicas, luchar contra la discriminación y la xenofobia, y el Pacto Social por la Inmigración en Euskadi habla de un reconocimiento formal de la no discriminación, que debe ir acompañado de actitudes y prácticas que favorezcan la igualdad, evitando prejuicios y todo tipo de trato discriminatorio. Pónganse los medios necesarios para ello.

\subsection{Dignidad}

Las diferentes políticas que hemos ido desgranando hasta ahora deben sustentarte bajo un pilar fundamental: la dignidad. El artículo I de la Declaración Universal de los Derechos Humanos dice que "todos los seres humanos nacen libres e iguales en dignidad y derechos, y dotados como están de razón y conciencia, deben comportarse fraternalmente los unos con los otros". No se pueden entender los derechos humanos sin la dignidad. La lucha para que no sean conculcados derechos básicos, como el de igualdad, salud física, acceso a un empleo y vivienda, es también la lucha por la dignidad de las personas.

Son numerosos los investigadores que apuntan a la dificultad de mantener una dignidad personal cuando no se tiene casa (Buckner, Bassuk y Zima, 1993; Miller y Keys, 2001; Seltser y Miller, 1993). El estigma social que acompaña a la situación sin hogar y las condiciones de degradación y deshumanización que conlleva pueden comprometer gravemente la dignidad de la persona que vive esa situación: "ser persona sin hogar afecta a la dignidad esencial del ser humano, deteriorando la capacidad de ser vistos y verse a sí mismos como personas valiosas" (Miller y Keys, 2001: 348-350). Reconocer la dignidad de una persona es reconocer su valor como ser humano, independientemente de su estatus o rol en la sociedad. La dignidad hace referencia a lo que somos, no a lo que tenemos. La experiencia de dignidad depende de cómo nos vemos nosotros mismos, y cómo somos vistos y tratados por los demás. La experiencia de dignidad es una interrelación de los individuos con su ambiente.

Cuando se hablaba del estigma, ya se ponía en valor la importancia de la mirada de la gente en el sentimiento de indignidad. La imagen que los demás proyectan de nosotros, junto con nuestra propia percepción de nuestro estado físico (suciedad, problemas físicos) y mental (la esperanza de poder salir de la situación en la que nos encontramos, el sentimiento de no importar a la sociedad). Son variables que están influyendo en que algunas personas se vean a sí mismas deshumanizadas, más cerca de los animales que de las personas. Se trata de una auténtica alienación, que provoca un mayor malestar psicológico y sufrimiento emocional, cambios en la 
identidad, cronificación de la vida en la calle y abuso de alcohol (Navarro, 2013).

Hay que situar esta problemática en sus justos términos, sin cargar la culpa en el propio individuo. Martín Baró (1998: 137) lo explica claro:

Cuando en psicología hablamos de alienación, solemos entender la pérdida de control que la persona sufre en relación con algún aspecto de su funcionamiento psicológico, y que le lleva a comportarse de manera considerada anormal. Pero la alienación es, primero y fundamentalmente, el estado que se genera en las personas a través de unas relaciones sociales que las expolian y despojan de su humanidad material y psíquica.

Dice el filósofo Avishai Margalit (2010:15) que "una sociedad decente es aquella que no humilla a las personas que se benefician de sus servicios. Una sociedad decente es aquélla en la que sus miembros no se humillan unos a otros". Ésa debe de ser la premisa sobre la que sustentemos las políticas e intervenciones con las personas sin hogar. La pregunta que se debe hacer siempre es: esta acción, este dispositivo, esta decisión, ¿dignifica a la persona o supone algún tipo de humillación?, ¿hace que pueda recuperar el control sobre su vida o la convierte en dependiente? Miller y Keys (2001) señalan algunos aspectos de los recursos asistenciales que pueden afectar a la dignidad de las personas, tales como ser tratados impersonal y negativamente por los trabajadores de los recursos, ser ignorados y evitados por la gente con la que se interactúa en la calle, la falta de privacidad en los lugares donde se 'habita', la existencia de normas excesivas y la 'institucionalización' de algunos recursos que imitan modelos de instituciones cerradas (cárceles y psiquiátricos). A ellos se les pueden unir otros, como la espera en lugares públicos para ser atendidos (muy en boga últimamente con los repartos de alimentos), las colas, los prejuicios y el trato desigual de algunos profesionales de la salud y la intervención social, el trato denigrante de algunos efectivos de los diferentes cuerpos de seguridad (policía local, Ertzaintza, vigilantes privados).

\subsection{Gestión del conocimiento y coordinación}

Hay, por último, dos aspectos transversales en el esquema planteado. El primero es la gestión del conocimiento. No es posible saber el efecto que tienen las políticas que se desarrollan si no se mide. Hasta ahora, la cuestión de las personas sin hogar apenas ha tenido importancia en el ámbito político y en el académico. Ejemplo de ello es que no hay una definición oficial de persona sin hogar en España y se adopta la proporcionada por Feantsa y promovida por las entidades sociales. Tampoco se han realizado estudios para conocer en profundidad el problema de las personas sin hogar y los únicos datos fiables a escala de comunidad autónoma son los de las encuestas del Eustat (2005 y 2012a). Esto está cambiando en los últimos años gracias a la colaboración de las entidades sociales y las instituciones públicas en los recuentos nocturnos. Se debe promover la creación, por parte de la Universidad, de un observatorio de las personas sin hogar que, en colaboración con las entidades sociales y las instituciones públicas, pueda recopilar datos, avanzar el conocimiento y evaluar los avances en la materia. Pero lo académico no se debe cerrar sobre sí mismo. Hay que promocionar la innovación social y la participación para, desde la propia acción, propiciar reflexiones que permitan un retorno a la intervención y una mejora de ésta.

La segunda línea transversal ya ha sido mencionada en el punto anterior: la coordinación. Contamos con un entramado institucional complejo y multinivel (ayuntamientos, diputaciones y Gobierno Vasco) que necesita marcar una hoja de ruta común, un reparto de tareas y funciones, y una coordinación constante en la ejecución. No es tarea nada fácil, porque a ello hay que añadir la coordinación entre las instituciones públicas y las entidades sociales, actor imprescindible en la lucha contra el sinhogarismo. Habrá que trabajar internamente también en la ruptura de los miedos y prejuicios existentes para el trabajo conjunto, así como en las cuestiones del poder. Todo ello sin perder de vista a Europa, es decir, las prácticas que nos pueden servir de ejemplo y las oportunidades (también financieras) que se abren con la Estrategia UE 2020.

\section{Conclusiones}

Lanzamos en el propio título del artículo una pregunta, ¿es posible acabar con el sinhogarismo en Euskadi o se trata de una quimera? A lo largo estas páginas, se ha intentado dar una panorámica lo más realista posible de la situación actual del fenómeno y las posibles políticas que se han de desarrollar. La respuesta a la pregunta inicial es, como en la mayoría de las preguntas, variable, pues depende fundamentalmente de la voluntad de hacerlo. Nos encontramos ante una buena oportunidad: en la Unión Europea, se han marcado unos objetivos claros y hay voluntad política para hacerlo; en Euskadi, contamos con algunos recursos en funcionamiento que pueden ser de gran ayuda (Etxebide, Lanbide) y otros con un potencial importante (desarrollo de la Ley de Servicios Sociales), y también hay un rico y variado tejido social que ha conseguido avances importantes en la lucha contra la exclusión social. Así pues, contamos con los elementos necesarios para poder responder afirmativamente a la pregunta: sí es factible acabar con el sinhogarismo en Euskadi y es un bonito objetivo que nos va a suponer el avance en otros muchos aspectos del bienestar social. Toca proponérselo en serio y trabajar por ello de manera coordinada. Estamos en el momento preciso, no podemos perder la oportunidad, la gente que peor lo pasa y la sociedad en su conjunto lo necesita. ¿Seremos capaces de hacerlo? 
(2012) "Real Decreto 1192/2012, de 3 de agosto, por el que se regula la condición de asegurado y de beneficiario a efectos de la asistencia sanitaria en España, con cargo a fondos públicos, a través del Sistema Nacional de Salud”, Boletín Oficial del Estado, ํㅜ 186, 4-8-2012, págs. 55.775-55.786 [<http://www.boe.es/diario_ boe/txt.php?id=BOE-A-2012-10477)].

(2011): “Ley 4/2011, de 24 de noviembre, de modificación de la Ley para la Garantía de Ingresos y para la Inclusión Social”, Boletín Oficial del País Vasco, no 233, 12-12-2011 [<http://www.bopv. euskadi.net/cgi-bin_k54/bopv_20?c\&f=201112 $12 \& a=201105924>$ '].

(2008): “Ley 12/2008, de Servicios Sociales del País Vasco", Boletín Oficial del País Vasco, n-2 246, 24-12-2008, págs. 31.840-31.924 [khttp://www.euskadi.net/ bopv2/datos/2008/12/0807143a.pdf)].

(2006) "Ley 2/2006, de 30 de junio, de Suelo y Urbanismo", Boletín Oficial del País Vasco, no 138, 20-72006, págs. 15.396-15.570 [<http://www.bopv. euskadi.net/cgi-bin_k54/bopv_20?c\&f=201112 $12 \& a=201105924$ ') .

(1998): “Ley 12/1998, de 22 de mayo, contra la exclusión social”, Boletín Oficial del País Vasco, n-105, 8-6-1998, págs. 10.467-10.506 [<http://www. bopv.euskadi.net/cgi-bin_k54/bopv_20?c\&f=1 9980608\&s=1998105'].

AIERDI, X. et al. (2009): Perfil y necesidades de las personas sin hogar en Bizkaia, Bilbao, Diputación Foral de Bizkaia.

ARARTEKO (2006): Respuesta a las necesidades básicas de las personas sin hogar y en exclusión grave, serie: Informes Extraordinarios, VitoriaGasteiz, Ararteko [/http://www.ararteko.net/ RecursosWeb/DOCUMENTOS/1/1_9_3.pdf)].
BAHR, H. M. (1973): Skid Row: An Introduction to Dissafiliation, Nueva York, Oxford University Press.

- (1968): Homelessness and Disaffiliation, Nueva York, Columbia University.

BLACKHART, G. C. et al. (2009): "Rejection elicits emotional reactions but neither causes immediate distress nor lowers self-esteem: A metaanalytic review of 192 studies on social exclusion", Personality and Social Psychology Review, vol. 13, nํㅜ 4, págs. 269-309.

BUCKNER, J. C.; BASSUK, E. L.; y ZIMA, B. T. (1993): “Mental health issues affecting homeless women: Implications for intervention", The American Journal of Orthopsychiatry, vol. 63, n- 3 , págs. 385-399.

CABRERA, P. (1998): Huéspedes del aire. Sociología de las personas sin hogar en Madrid, Madrid, Universidad Pontificia de Comillas.

CABRERA, P.; y RUBIO, M. J. (2008): "Las personas sin hogar, hoy", Revista del Ministerio de Trabajo e Inmigración, ํㅜ 75, págs. 51-74.

CABRERA, P.; RUBIO, M. J.; y BLASCO, J. (2008): ¿Quién duerme en la calle? Una investigación social y ciudadana sobre las personas sin techo, Barcelona, Fundació Caixa Catalunya.

COMISIÓN ONARTU (2011): Informe técnico sobre la situación de las personas sin hogary los recursos de ámbito municipal y foral, Bilbao, Ayuntamiento de Bilbao.

COMISIÓN EUROPEA (2010): Propuesta de informe conjunto sobre protección social e inclusión social, Bruselas, Comisión Europea, COM(2010)25 Final.

CONSEJO DE EUROPA (1996): Carta social europea (revisada), Estrasburgo, Consejo de Europa. 
- (1995): Protocolo adicional a la carta social europea que prevé un sistema de reclamaciones colectivas, Estrasburgo, Consejo de Europa.

DUQUE, J. M. (2012): “Redescubrimiento de los servicios sociales de atención primaria: hacia un (nuevo) modelo de atención personal y comunitaria", Zerbitzuan, nํ52, págs. 23-44 [khttp://dx.doi. org/10.5569/1134-7147.52.02>].

EPEL, E. S.; BANDURA, A.; y ZIMBARDO, P. G. (1999): "Escaping homelessness: The influences of self-efficacy and time perspective on coping with homelessness", Journal of Applied Social Psychology, vol. 29, n- 3, págs. 575-596.

EUSTAT (2012a): Encuesta sobre Personas sin Hogar 2012, Vitoria-Gasteiz, Eustat [<http://www.eustat.es/ estadisticas/tema_219/opt_o/ti_Personas_ sin_hogar/temas.html\#axzz2kRxs1uJ5>].

- (2012b): Estadística de Recursos para Personas sin Hogar Vitoria-Gasteiz, Eustat [<http://www.eustat.es/ estadisticas/tema_219/opt_o/ti_Personas_ sin_hogar/temas.html\#axzz2kRxs1uJ5>].

- (2012c): Estadística sobre Vivienda Vacía, VitoriaGasteiz, Eustat [http://www.eustat.es/ estadisticas/tema_264/opt_1/ti_Estadistica_ sobre_vivienda_vacia_EVV/temas. html\#axzz2lovDrpSe>].

- (2005): Encuesta sobre Personas sin Hogar 2005, VitoriaGasteiz, Eustat.

FACIAM (2010): 10 Propuestas para una estrategia de erradicación del sinhogarismo, Federación de Asociaciones de Centros para la Integración y Ayuda de Marginados [<http://faciam. org/especiales/presentacion_decalogo/ presentacion_decalogo.html s].

FAZEL, S. et al. (2008): "The prevalence of mental disorders among the homeless in western countries: Systematic review and meta-regression analysis", PLoS Medicine, vol. 5, $\mathrm{n}$ - 12, e225 [rhttp://dx.doi.org/10.1371/journal. pmed.0050225'].

FEANTSA (2013): Health and Well-being for All - Holistic Health Services for People Who Are Homeless, Bruselas, European Federation of National Organisations Working with the Homeless [<http://www.feantsa.org/spip. php?article385\&lang=en>].

- (2008): The Role of Housing in Pathways into and out of Homelessness. Annual Theme 2008. Housing And Homelessness, Bruselas, European Federation of National Organisations Working with the Homeless.

- (2006): FEANTSA Annual European Report 2006. The Right to Health is a Human Right: Ensuring Access to Health for People Who are Homeless, Bruselas, European Federation of National Organisations Working with the Homeless.

- (2005): ETHOS - European Typology of Homelessness and Housing Exclusion, Bruselas, European Federation of National Organisations Working with the Homeless [<http://www.feantsa.org/ spip.php?article120\&lang $=e n>]$.

FITZPATRICK, S.; KEMP, P.; y KLINKER, S. (2000): Single Homelessness: An Overview of Research in Britain, Bristol, The Policy Press; University of Bristol.
GARAY, M. et al. (2012): “Evolución del Programa de Asistencia Psiquiátrica a Personas Sin Hogar con Enfermedad Mental Grave en el municipio de Bilbao", Zerbitzuan, no 52 , págs. 179-190 [khttp://dx.doi.org/10.5569/1134-7147.52.12〉].

GOBIERNO VASCO, Departamento de Vivienda, Obras Públicas y Transportes (2010): Pacto Social por la Vivienda. Un acuerdo para construir futuro, Vitoria-Gasteiz, Servicio Central de Publicaciones del Gobierno Vasco [rhttp:// www6.euskadi.net/r41-430/es/contenidos/ noticia/pacto_social_vivienda/es_psv/ adjuntos/psv.pdf〉].

GOFFMAN, E. (1980): Estigma: la identidad deteriorada, Buenos Aires, Amorrortu.

LEHMAN, A. F.; y CORDRAY, D. S. (1993): “Prevalence of alcohol, drug and mental disorders among the homeless: One more time", Contemporary Drug Problems, vol. 20, págs. 355-383.

MARGALIT, A. (2010): La sociedad decente, Barcelona, Paidós.

MARTÍN-BARÓ, I. (1988): “La violencia política y la guerra como causas del trauma psicosocial en El Salvador", Revista de Psicología de El Salvador, vol. 7, págs. 123-141.

MILLER, A. B.; y KEYS, C. B. (2001): "Understanding dignity in the lives of homeless persons", American Journal of Community Psychology, vol. 29, nํㅜ 2, págs. 331-354.

MUÑOZ, M.; VÁZQUEZ, C.; y VÁZQUEZ, J. J. (2003): LoS límites de la exclusión: estudio sobre los factores económicos psicosociales y de salud que afectan a las personas sin hogar en Madrid, Madrid, Caja Madrid; Ediciones Témpora.

NACIONES UNIDAS (1948): Declaración Universal de Derechos Humanos [khttp://www.un.org/es/ documents/udhr/s].

NACIONES UNIDAS, Consejo Económico y Social, Comité de Derechos Económicos, Sociales y Culturales (2004): Observaciones finales del Comité de Derechos Económicos, Sociales y Culturales: Spain, 07/06/2004, E/C.12/1/Add.99 [rhttp:// www.unhchr.ch/tbs/doc.nsf/\%28Symbol\%29/ E.C.12.1.Add.99.Sp?Opendocument $>$.

NAVARRO, M. A. (2013): "Análisis psicosocial de la exclusión residencial extrema en la población migrante" [tesis doctoral], Madrid, Universidad Pontifica de Comillas.

OBSERVATORIO VASCO DE LA VIVIENDA (2013a): Fórmulas alternativas a la compra y el alquiler en el acceso a la vivienda, Vitoria-Gasteiz, Observatorio Vasco de la Vivienda [khttp://www.garraioak. ejgv.euskadi.net/contenidos/informacion/ ovv_sv_alternativas/es_ovv_sevi/adjuntos/ Alternativas\%20a\%2ola\%20compra\%20y\%20 alquiler\%2ode\%2ovivienda.pdf $\rangle]$.

- (2013b): Informe de evaluación de la política de alquiler en 2012, Vitoria-Gasteiz, Observatorio Vasco de la Vivienda [<http://euskadi.net/ r41-ovado3/es/contenidos/informacion/ ovv_adm_alquiler2012/es_ovv_admi/ovv_ administracion161_es.html >].

- (2012): Breve análisis sobre la evolución de la vivienda vacía en la CAE 1997-2011, Vitoria-Gasteiz, 
Observatorio Vasco de la Vivienda [<http:// euskadi.net/r41-ovse03/es/contenidos/ informacion/ovv_sectorvivienda_vv/es_ovv_ sevi/ovv_sectorvivienda213_es.htmlı].

PARLAMENTO EUROPEO (2010): Estrategia de la UE en relación a las personas sin hogar, Estrasburgo, Parlamento Europeo, P7_TA(2010)0499.

ROBERTSON, M. J. (1992): "The prevalence of mental disorders among homeless people", en JAHIEL, R. I. (ed.), Homelessness, Nueva York, John Hopkins Univsersity Press.

ROSSI, P. H. (1989): Down and Out in America. The Origins of Homelessness, Chicago, University Chicago Press.

SELTSER, B. J.; y MILLER, D. E. (1993): Homeless Families: The Struggle for Dignity, Urbana, University of Illinois Press.

SIIS CENTRO DE DOCUMENTACIÓN Y ESTUDIOS (2013): Estudio sobre la situación de las personas en situación de exclusión residencial grave en la CAPV, Vitoria-Gasteiz, Eusko Jaurlaritza-
Gobierno Vasco, Departamento de Empleo y Asuntos Sociales [<http://www.siis.net/es/ descarga-informes. php?id=257>].

SOS RACISMO; y CEAR-EUSKADI (2012): “Práctica del testing en el estudio de la discriminación en el acceso a la vivienda de la población migrante y refugiada en Bilbao", Mugak, $\mathrm{n} \stackrel{0}{58}$, págs. 38-41.

TAYLOR, C. (1993): "On the conduct of homelessness research: Lessons from a qualitative study of women diagnose with chronic mental illness", Issues in Mental Health Nursing, $\mathrm{n}-14$, págs. 432-453.

VALVERDE, J. (2009): “Apuntes sobre el diálogo terapéutico en la intervención sobre personas en situación de exclusión social”, en MARKEZ, I.; FERNÁNDEZ, A.; y PÉREZ-SALES, P. (eds.), Violencia y salud mental, Madrid, Asociación Española de Neuropsiquiatría.

WRIGHT, J. D.; y WEBER, E. (1987): Homeless and Health, Nueva York, McGraw-Hill. 